\title{
Zum ästhetischen Wertewandel in Kultur und Kosmetik
}

\author{
C. Wietig \\ S. Williams \\ T. Reuther \\ M. Davids \\ M. Kerscher
}

\author{
The Changing Face of Aesthetic Ideals in Cosmetics and Culture
}

\section{Zusammenfassung}

Die Schönheitspflege dient seit jeher dem Mythos Alterslosigkeit. Moderne dermatokosmetische Behandlungsverfahren und ästhetisch-chirurgische Korrekturen vermögen den bisher mehr oder minder schicksalhaft verlaufenden, sichtbaren Alterungsprozess aufzuhalten bzw. zu verzögern. „Schönheit“ als Kompositum aus Gesundheit, Jugendlichkeit und sexueller Attraktivität gewinnt in der evidenzbasierten kosmetischen Dermatologie, der auf wissenschaftlichen Erkenntnissen beruhenden Kosmetologie, zunehmend an Bedeutung. Als Wiege kosmetischer Behandlungen gilt das antike Ägypten. Im Papyros Ebers (1552 v. Chr.), dem wahrscheinlich ältesten bekannten hermetischen Zeugnis, stehen medizinische und kosmetische Rezepturen gleichberechtigt nebeneinander. Eine Trennung kosmetischer von medizinisch-dermatologischen Maßnahmen gab es in dieser Zeit nicht. Der französische Chirurg Henri de Mondeville (1260-1320 n. Chr.) gilt als einer der ersten Ärzte, die zwischen medizinischen und kosmetischen Maßnahmen unterschieden. Der moderne kosmetisch-medizinische Ansatz knüpft in gewissen Aspekten an die Tradition der ganzheitlichen Sichtweise an, jedoch unter veränderten Vorzeichen medizinischer Hochtechnologie der Life-Sciences. Da der Körper kosmetisch modifiziert immer auch als Kulturträger seiner Zeit fungiert, drückt die jeweilige Körperbildästhetik auch zeitimmanente Konventionen aus. Vor dem Hintergrund einer international zunehmenden Nachfrage nach dermatokosmetischen Maßnahmen stellt der Artikel eine Auswahl kulturgeschichtlicher Aspekte der „Schönerhaltung“ des Körpers im Wandel der Zeit dar.

\section{Abstract}

Skin care and aesthetic treatments, which aim for the myth of eternal 'no-age', have been performed for many centuries. While modern dermatocosmetic formulations and aesthetic procedures are able to ameliorate or delay the development of visible signs of ageing to a certain degree, the ageing process itself is inevitable. 'Beauty' as a combination of health, youth and sexual attraction, is receiving increasing interest and consideration in evidence-based cosmetic dermatology, the scientifically proven cosmetic dermatology. Ancient Egypt is seen as the birthplace of cosmetic treatments. The oldest known testimony of cosmetic skin care formulations is the 'Papyros Ebers' (1552 BC), which contains both medical and cosmetic formulations. A strict separation of medical-dermatologic therapy on the one hand and cosmetic measures on the other hand had been unknown for many centuries. The French surgeon Henri de Mondeville (1260-1320 $\mathrm{AD}$ ) is thought to be one of the first physicians who separated medical treatments from cosmetic treatments. The modern combination of cosmetic and medical dermatology thus returns in some way to traditional aspects of cosmetic treatment methods. Today's aesthetic-cosmetic medicine however represents a hightech life-science. The cosmetically modified body has always been a mirror of the culture of the time. Having the increasing demand for dermatocosmetic treatments in mind, the article describes cultural aspects of the changing face of aesthetic ideals. 
Die Haut des Menschen als originäre, schützende Hülle des Ichs wird seit Menschengedenken dem jeweiligen Kulturkreis entsprechend modifiziert und den derzeit herrschenden Schönheitsidealen mit dem Ziel sozialer Aufwertung angepasst $[13,22,32,3,5,25]$. Das perfekte Körperbild wird seit der Antike von vielen Menschen angestrebt, nicht zuletzt da die „Schönheit“ auch soziale Macht beinhaltet. Schon vor Jahrhunderten wurde ein „strahlender“ Teint durch diverse pflegerische, dekorative und therapeutische Maßnahmen angestrebt. Eine glatte Oberfläche reflektiert physikalisch mehr Licht, so dass die Haut zart glänzt und damit Makellosigkeit, die mit göttlicher Ausstrahlung gleichgesetzt wurde, symbolisierte [10]. Religions-, philosophie-, literatur- und kunstgeschichtliche Annäherungen an das „Unbenennbare des ewigen Schönen“, das universell Anerkannte, dem die Unsterblichkeit immanent ist, berühren die Ebene des Sakralen und Unantastbaren [35,1,17,31]. Schönheitspflege und Mythos Alterslosigkeit sind daher untrennbar, womit sich der Schönheitskult auf Gesunderhaltung und dekorativer Schönerhaltung gründet [37]. Der immerwährende Modewandel der Schönheitsideale zeugt vom ästhetischen Wertewandel der Körperbildästhetik in Kultur und Kosmetik.

Die Dermatokosmetik dokumentiert entwicklungsgeschichtlich auch den kulturellen Prozess von magischen zu naturwissenschaftlichen Behandlungstechniken $[30,40]$. In bestimmten Aspekten greift die moderne ästhetisch-kosmetische Medizin eine bereits in der Antike praktizierte Kombination von medizinischen und kosmetischen Behandlungsverfahren wieder auf, heute jedoch mit evidenzbasierten Methoden und High-techVerfahren. Moderne dermatokosmetische Behandlungsverfahren und ästhetisch-chirurgische Korrekturen vermögen den bisher mehr oder minder schicksalhaft verlaufenden, sichtbaren Alterungsprozess aufzuhalten bzw. zu verzögern [33]. Eine Annäherung an das ideale Körperbild ist daher im Computerzeitalter der Life-Sciences zu gewissen Teilen konstruierbar und kann als Ware erworben werden. Unser Aussehen wird zukünftig zunehmend technisch-prothetisch, biotechnologisch oder vielleicht sogar gentechnisch verändert, verbessert und „designed“ werden können. Der Begriff „Schönheit“ als Kompositum aus Gesundheit, Jugendlichkeit und sexueller Attraktivität gewinnt auch in der evidenzbasierten kosmetischen Dermatologie, der auf wissenschaftlichen Erkenntnissen beruhenden kosmetischen Dermatologie, zunehmend an Bedeutung. Aktuelle Entwicklungen in der Dermatologie tragen dem stetig größer werdenden Bedürfnis nach präventiven wie auch therapeutischen Maßnahmen für ein jüngeres, attraktiveres und gesünderes Aussehen Rechnung. Dies zeigt sich zum Beispiel eindrucksvoll an der nahezu exponentiell steigenden Anzahl an Publikationen zum Einsatz von Botulinumtoxin in der ästhetischen Dermatologie (Abb.1).

Körpergefühl, Sauberkeitsbedürfnisse und Schönheitsempfinden gehören zum einen in den Bereich des individuellen Erlebens, repräsentieren auf der anderen Seite jedoch auch einen gesellschaftlichen Code. So fungiert der Körper als Kulturträger und ästhetischer Ort der Erfahrung seiner jeweiligen Zeit und drückt Fixierungen der jeweils technisch-realisierbaren Ästhetik aus. Davon zeugen zum Beispiel Ansätze kosmetischer Körperkultur wie auch das Spektrum der Hygienegeschichte durch die Jahrhunderte bis hin zur abendländischen Reinlichkeitskultur $[1,28,41,38,18,26,40]$. Nachfolgend soll ein kleiner Überblick in

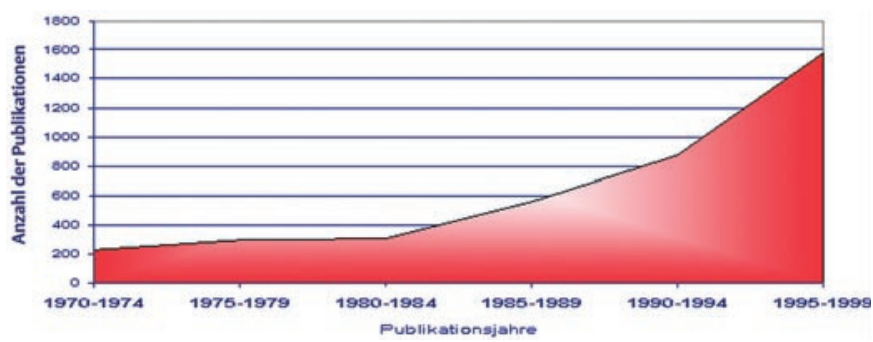

Abb. 1 Publikationen zum Einsatz von Botulinumtoxin in der ästhetischen Dermatologie.

Aspekte ausgewählter Epochen bis hin zur Gegenwart den Stellenwert kosmetischer Behandlungen beispielhaft dokumentieren (Abb. 2).

Die antiken Ägypter suchten nach ewiger Schönheit und Vollkommenheit für Lebende wie auch Tote [11]. Die Technik der Mumifizierung steht in direkter Wechselbeziehung mit der Verschönerung der Lebenden und trug auch zur Wissensvermehrung auf dem medizinischen Sektor bei [7]. Bereits hier werden die Anfänge des Mythos Alterslosigkeit gelegt [40]. Der lebende wie auch der tote Körper wurde mit den Schminken der antiken Ägypter verschönert und geschützt. Die Schminken dienten auch als Grabbeigaben und gehörten beim Bau eines Tempels - neben anderen Opfergaben - als rituelle Grundsteinbeigaben unter die Ecken der Außenmauern oder den Eingang [30]. Im Papyros Ebers, einer vollständig erhaltenen medizinischen Textsammlung der antiken Ägypter aus dem Jahr 1552 v. Chr., stehen zahlreiche medizinische und kosmetische Rezepturen gleichberechtigt nebeneinander [29]. Eine Trennung von kosmetischen und medizinisch-dermatologischen Maßnahmen gab es unter diesem synkretistischen Ansatz nicht.

Im scholastischen Weltbild wurde die Schönerhaltung des Körpers unter dem Vanitasaspekt idealisiert und in eine Erhöhung der „schönen Seele“ transzendiert [6]. Das Geistige überdeckte in dieser Zeit das Weltliche, und der Begriff des „Fleisches“ erfuhr eine Abwertung durch das Christentum, weil es mit niedriger, sündiger Triebhaftigkeit besetzt wurde [34]. Dies blieb nicht ohne Folgen auf den Bereich der Körperpflege, die nunmehr fast ausschließlich auf Reinlichkeit und Sauberkeit zielte und symbolisch auch einer Reinwaschung der Seele diente. Die Anwendung pflegender Externa geriet unter den Verdacht der „Teufelsbuhlschaft" um der Eitelkeit willen. Dekorative Kosmetik bedeutete aus orthodoxer Sicht eine weitere Verleugnung. „Eitle“, die zum Beispiel das Übel des Haarausfalles dennoch nicht ertragen wollten, konnten jedoch auf diverse alchemistisch anmutende kosmetische Mittel, etwa zur Haarwuchsförderung, zurückgreifen [20].

Die Körperpflegekultur des Mittelalters basierte auf den balneologischen Erfahrungen der Antike, der Humoralpathologie sowie dem „Analogiezaubern“, deren Wurzeln auch in der christlichideologischen Reinwaschung liegen. Schwitzbäder, Aderlass und die Reinigung der Haut dienten der Gesunderhaltung [23]. Bademägde, Bader und Barbierchirurgen übten auch eine soziale Kontrolle über das Hauterscheinungsbild aus. Der Tätigkeitsbereich der Barbierchirurgen verdeutlicht zudem, dass in dieser Zeit 




Abb. 2 Entwicklungsgeschichte der Kosmetik.

3.-4. Jh. n. Chr.

noch keine klare Trennung zwischen medizinischer und kosmetischer Versorgung bestand. So führten die Barbierchirurgen neben Haarschneiden und Rasieren auch die niedere Wundmedizin aus [38].

Zu einem ästhetischen Wertewandel kam es durch den kulturellen Austausch mit dem Orient durch die Kreuzzüge vom Ende des 11. bis Ende des 13. Jahrhunderts. Dieser kulturelle Austausch trug in Europa zu einer wieder mehr sinnenfreudigen Diesseitigkeit bei. Er kultivierte auch die europäische Ritterschaft und verfeinerte die Sitten [16]. Eine herausragende Persönlichkeit des Mittelalters war der französische Hofchirurg Henri de Mondeville (1260 - 1320), der als einer der ersten Ärzte gilt, der zwischen medizinischen Maßnahmen auf der einen Seite und kosmetischen Maßnahmen zur Verbesserung des Äußeren auf der anderen Seite unterschied [39]. Er führte 1315 auch erstmalig Sektionen an Frauen aus.

Die auf antiken Schönheitsidealen basierende Körperbildästhetik des Humanismus förderte die Neubewertung subjektiver Körperpflegemaßnahmen des Individuums. Der Körper fungierte als Medium, sowohl als Ort ästhetischer Erfahrungen wie auch gesellschaftlicher Stellung $[14,8,4]$. So bezogen sozial hoch gestellte Damen ihre Kosmetikprodukte bevorzugt aus Venedig und experimentierten mit ihren persönlichen Mixturen, Cremes, Seifen und Parfums. Caterina Sforza (1462-1509) zum Beispiel hat in ihren Experimenti auch Rezepturen zum Bleichen der Haare hinterlassen $[20,31]$. Die bekannte Gesundheitsschädlichkeit der dekorativen Schminken wurde für die standesabgrenzende Distinktion in Kauf genommen. So wurde zum Beispiel in der Renaissance dem Ideal einer strahlend weißen Haut mit reichlich arsen- oder quecksilberhaltigem Bleiweiß nachgeholfen, obschon die verheerende hautschädigende Wirkung bis hin zur letalen Intoxikation leidvoll erfahren wurde [27].

Die naturwissenschaftliche Auseinandersetzung mit dem Körper begründete das 1543 veröffentliche Werk De Humani Corporis Fabrica Libri Septem des flämischen Arztes Andreas Vesalius
(1514 - 1564). Obschon es jedoch eine medizinisch-naturwissenschaftliche Sichtweise förderte, verhafteten die Rezepturen für die kosmetische Schönerhaltung des Körpers größtenteils noch in der Magie der Analogzauber.

Im Zeitalter der „trockenen Toilette“ des Barock wurden in der höfischen Gesellschaft geschlechter- und altersübergreifend Puder, Pomaden, Schminken, stark duftende Parfums, Abreibungen mit Wein oder Essig und häufiger Wäschewechsel dem Waschen mit Wasser vorgezogen. Wasser wurde nur sparsam, etwa für die Reinigung der Leibwäsche, verwendet. Dies war in der Zeit der Fäkalgruben - vor der Schwemmkanalisation - eine sinnvolle Vorsichtsmaßnahme [41,12]. Ebenso wurde die „vornehme Blässe" der Renaissance tradiert und kosmetische Maßnahmen als Zeichen der Distinktion politisch instrumentalisiert und weiter aufgewertet [9]. Die Überformung der Natürlichkeit durch Künstlichkeit entsprach dem cartesianischen Zeitgeist und symbolisierte Überhöhung [15]. Die kosmetischen Maßnahmen der höfischen Gesellschaft, die vor der Demokratisierung der Mode Vorbildcharakter hatten, erreichten im Barock und Rokoko ihren Höhepunkt [36,9].

Im Zeitalter der Aufklärung erlangte die Schädlichkeit der aristokratischen Schminken durch Kritik von Medizinern zunehmende Aufmerksamkeit [21]. Ratgeber ihrer Zeit empfahlen, natürliche Schönheit zu unterstützen, und stärkten so auch die Wertvorstellungen des erstarkenden Bürgertums [2]. Sauberkeit und Anstand begründeten das neue Hygieneverständnis im 19. Jahrhundert, das didaktisch zur Bürgerpflicht erhoben wurde [26]. Die Qualität der verwendeten Kosmetikgüter unterschied die sozialen Klassen [19]. Die aufkommende Sportbewegung sowie der Körper- und Lichtkult setzten zum Ende des 19. Jahrhunderts neue Akzente.

Im Jahre 1912 eröffnete Helena Rubinstein ihren ersten Schönheitssalon in Paris. Sie übernahm für ihre kosmetischen Entwicklungen als Erste die wissenschaftliche Terminologie und bildete damit bereits eine Brücke zur modernen kosmetischen Medizin. 
In den folgenden Jahrzehnten hielten Laborentwicklungen, die Entstehung von Schönheitsinstituten, eine sich vermehrt durchsetzende plastische Chirurgie, Augmentationstechniken, etwa die Injektion von Paraffin in die Wangen sowie die ersten Antifalten-Cremes, etwa die 1969 aufkommenden, Kollagen enthaltenden Kosmetika, den Wettstreit der Forschung im Fluss und bereiteten somit den Boden für die moderne kosmetische Dermatologie [32]. Nachdem zunächst vor allem Frauen Marketingziel der großen Kosmetikfirmen waren, wurden in den 80er-Jahren auch Männer für den Kosmetikmarkt entdeckt und seither zunehmend angesprochen. Heute wird eine Annäherung an individuelle und gesellschaftlich geprägte Körperideale durch innovative dermatokosmetische Zubereitungen und Verfahren, plastische Chirurgie und medizinisch-kontrollierte Gewichtsreduktion vermehrt machbar. Der ästhetische Wertewandel in Kultur und Dermatokosmetik bewirkt heute auch eine veränderte Bewertung zeitimmanenter Technologien, etwa moderner Lasergeräte, die nicht nur der medizinischen Behandlung, sondern auch einer Schönerhaltung des Körpers dienen können. Nach aktuellen Erkenntnissen folgt dem Bedürfnis nach einem gesunden Hautorgan unmittelbar der Wunsch nach einer schönen Haut mit jugendlichem Erscheinungsbild. In unserer modernen Signalkultur verspricht das steigende Bedürfnis nach Umgestaltung der Haut der kosmetischen Industrie wie auch der kosmetischen Medizin erfolgreiche Entwicklungsmöglichkeiten. Zusammenfassend stellt die evidenzbasierte kosmetische Dermatologie eine Erweiterung des mehr auf dekorativen Aspekten beschränkten traditionellen Begriffs der Kosmetik dar.

\section{Literatur}

${ }^{1}$ Angeloglou M. A history of make-up. London: Studio Vista Limited, Butler \& Tanner Limited, 1970

2 . Anmuth und Schönheit aus den Misterien der Natur und Kunst für ledige und verheirathete Frauenzimmer mit Kupfern, Berlin 1797 Reprint der bibliophilen Taschenbücher. Dortmund: Harenberg Kommunikation, 1978

${ }^{3}$ Anzieu D. Das Haut-Ich. Frankfurt a. M: Suhrkamp Taschenbuch Wissenschaft, Suhrkamp Verlag, 1991

${ }^{4}$ Belting H. Bild-Anthropologie, Entwürfe für eine Bildwissenschaft. München: Wilhelm Fink Verlag, 2001

${ }^{5}$ Benthien C. Haut. Literaturgeschichte - Körperbilder - Grenzdiskurse. Hamburg: Rowohlts Enzyklopädie, Rowohlt Taschenbuch, 1999

${ }^{6}$ Bernhard von Clairvaux . Sermones super Cantica Canticorum In: Eco U. Kunst und Schönheit im Mittelalter. München: dtv Wissenschaft, 1995

${ }^{7}$ Brandenburg D. Geschichte der Dermatologie. Mumien-Kosmetik vom Altertum bis heute. Ärztliche Kosmetologie 1985; 15, 3: 205-214

${ }^{8}$ Burckhardt J. Die Kultur der Renaissance in Italien. Herrsching: Pawlak, 1981

9 . Vom ewigen Zwang zu gefallen, Etikette und äußere Erscheinung. Burgelin O (Hrsg.). Leipzig: Reclam, 1994

${ }^{10}$ Campsie J, Craymer C. Modern life - Strahlender Teint. Köln: Könemann, 2001

${ }^{11}$ Champdor A. Das ägyptische Totenbuch in Bild und Deutung, bearbeitet von Manfred Lurker. Bindlach: Gondrom, 1992

${ }^{12}$ Corbin A. Pesthauch und Blütenduft, Eine Geschichte des Geruchs. Berlin: Wagenbach, 1984

${ }^{13}$ Erfindung des Menschen. Schöpfungsträume und Körperbilder 1500 - 2000. Dülmen R v (Hrsg). Wien, Köln, Weimar: Böhlau, 1998

${ }^{14}$ Dülmen R v. Die Entdeckung des Individuums, 1500-1800. Frankfurt a. M: Fischer Taschenbuch Verlag, 2002
${ }^{15}$ Elias N. Die höfische Gesellschaft, Untersuchungen zur Soziologie des Königtums und der höfischen Aristokratie. Darmstadt: Suhrkamp, 1983

${ }^{16}$ Elias N. Über den Prozeß der Zivilisation, Soziogenetische und psychogenetische Untersuchungen. Frankfurt a. M: Suhrkamp, 1995

${ }^{17}$ Frazer JG. Der goldene Zweig, Das Geheimnis von Glauben und Sitten der Völker, Kulturen \& Ideen. Hamburg: Rowohlts Enzyklopädie, 1989

${ }^{18}$ Gosman U. Sind Sie ganz sauber? Körperkult als Seifenoper. Psychologie Heute, August 1996; 8: 64-67

${ }^{19}$ Haubl R. „Unter lauter Spiegelbildern...“, Zur Kulturgeschichte des Spiegels. Frankfurt a. M: Nexus, 1991

${ }^{20}$ Die Frisur, Eine Kulturgeschichte der Haarmode von der Antike bis zur Gegenwart veranschaulicht an Kunstobjekten der Sammlung Schwarzkopf und internationaler Museen. Jedding-Gesterling M (Hrsg.). Neumünster: Wachholtz, 1988

21 Jedding-Gesterling M. Vom Rokokokavalier zum „echten Mann“. In: Sehnsucht nach Vollkommenheit, Die Sammlung Schwarzkopf im neuen Licht im Deutschen Hygiene-Museum. Darmstadt: Die Initiative, 1995

22 Jockel N. Nackt. Ästhetik der Blöße. München, London, New York: Prestel, 2002

${ }^{23}$ Kiby U. Bäder und Badekultur im Orient und Okzident, Antike bis Spätbarock. Köln: Dumont, 1995

${ }^{24}$ Klein G. Der Körper als Erfindung. In: Randow, Gero von (Hrsg.). Wie viel Körper braucht der Mensch. Standpunkte zur Debatte für den Deutschen Studienpreis. Hamburg: Edition Körber-Stiftung, 2001

25 Kunstforum. Band 141, Die oberflächlichen Hüllen des Selbst, Mode als ästhetisch-medialer Komplex. Kunstforum International, JuliSeptember 1998

${ }^{26}$ Labisch A. Homo Hygienicus, Gesundheit und Medizin in der Neuzeit. Frankfurt a. M., New York: Campus, 1992

${ }^{27}$ Liggett A, Liggett J. Die Tyrannei der Schönheit. München: Heyne, 1990

${ }^{28}$ Lohse-Jasper R. Die Farben der Schönheit. Eine Kulturgeschichte der Schminkkunst. Hildesheim: Gerstenberg, 2000

29 Papyros Ebers, Das hermetische Buch über die Arzneimittel der Alten Ägypter in hieratischer Schrift, Leipzig 1875. Osnabrück: Biblio Verlag, 1987

${ }^{30}$ Paszthory E. Salben, Schminken und Parfüme im Altertum, Herstellungsmethoden und Anwendungsbereiche im östlichen Mediterraneum. Antike Welt, Zeitschrift für Archäologie und Kulturgeschichte Sondernummer 1990; 21. Jahrgang:

${ }^{31}$ Rimmel E. Das Buch des Parfums, Die klassische Geschichte des Parfums und der Toilette. Dreieich: Hesse \& Becker, 1985

32 Schefer Faux D. Schönheit, Beauty, Beauté. Eine Kulturgeschichte des 20. Jahrhunderts. München: Schirmer/Mosel, 2000

${ }^{33}$ Schlich T. Eine kurze Geschichte der Körperverbesserung. In: Randow, Gero von (Hrsg.). Wie viel Körper braucht der Mensch. Standpunkte zur Debatte für den Deutschen Studienpreis. Hamburg: edition Körber-Stiftung, 2001

${ }^{34}$ Schmolke-Hasselmann B. „Camuse Chose“. Das Häßliche als ästhetisches und menschliches Problem in der altfranzösischen Literatur. In: Zimmermann, A (Hrsg). Die Mächte des Guten und des Bösen. Bd. 11. Berlin, New York: Veröffentlichungen des Thomas-Instituts der Universität zu Köln, 1977

${ }^{35}$ Schoske S, Grimm A, Kreißl B. Schönheit, Abglanz der Göttlichkeit, Kosmetik im Alten Ägypten. München: Lipp, 1990

${ }^{36}$ Schultz A. Alltagsleben einer deutschen Frau zu Anfang des 18. Jahrhunderts. Leipzig: Verlag S. Hirzel, 1890

37 Sehnsucht nach Vollkommenheit, Die Sammlung Schwarzkopf im neuen Licht im Deutschen Hygiene-Museum Darmstadt: Die Initiative, 1995

${ }^{38}$ Stolz S. Die Handwerke des Körpers, Bader, Barbier, Perückenmacher, Friseur. Folge und Ausdruck historischen Körperverständnisses. Marburg: Jonas Verlag, 1992

${ }^{39}$ Toellner R. Illustrierte Geschichte der Medizin. Salzburg: Andreas \& Andreas Verlagsbuchhandel, 1986

${ }^{40}$ Kosmetik, Entwicklung, Herstellung und Anwendung kosmetischer Mittel. Umbach W (Hrsg.). Stuttgart, New York: Thieme, 1995

${ }^{41}$ Vigarello G. Wasser und Seife, Puder und Parfüm, Geschichte der Körperhygiene seit dem Mittelalter. Frankfurt a. M., New York: Campus, 1992 